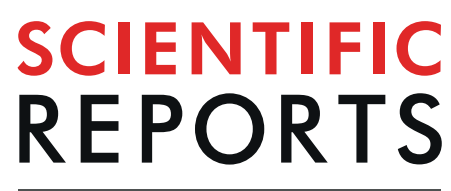

natureresearch

\title{
Tunable High Spatio-Spectral Purity Undulator Radiation from a Transported Laser Plasma Accelerated Electron Beam
}

\author{
A. Ghaith ${ }^{1,2^{*}}$, D. Oumbarek ${ }^{1,2}$, E. Roussel ${ }^{3}$, S. Corde ${ }^{4}$, M. Labat $^{1}$, T. André $^{1,2}{ }^{2}$ A. Loulergue ${ }^{1}$, \\ I. A. Andriyash ${ }^{5}$, O. Chubar 6 , O. Kononenko ${ }^{4}$, S. Smartsev ${ }^{4,5}$, O. Marcouillé ${ }^{1}$, C. Kitégi ${ }^{1}$, \\ F. Marteau ${ }^{1}$, M. Valléau ${ }^{1}$, C. Thaury ${ }^{4}$, J. Gautier ${ }^{4}$, S. Sebban ${ }^{4}$, A. Tafzi ${ }^{4}$, F. Blache ${ }^{1}$, F. Briquez ${ }^{1}$, \\ K. Tavakoli ${ }^{1}$, A. Carcy ${ }^{1}$, F. Bouvet ${ }^{1}$, Y. Dietrich ${ }^{1}$, G. Lambert ${ }^{4}$, N. Hubert ${ }^{1}$, M. El Ajjouri ${ }^{1}$, \\ F. Polack ${ }^{1}$, D. Dennetière ${ }^{1}$, N. Leclercq ${ }^{1}$, P. Rommeluère ${ }^{1}$, J.-P. Duval ${ }^{1}$, M. Sebdaoui ${ }^{1}$, \\ C. Bourgoin ${ }^{1}$, A. Lestrade ${ }^{1}$, C. Benabderrahmane ${ }^{1}$, J. Vétéran ${ }^{1}$, P. Berteaud ${ }^{1}$, C. De Oliveira ${ }^{1}$, \\ J. P. Goddet ${ }^{4}$, C. Herbeaux ${ }^{1}$, C. Szwaj ${ }^{3}$, S. Bielawski ${ }^{3}$, V. Malka ${ }^{4,5}$ \& M.-E. Couprie ${ }^{1,2}$
}

Undulator based synchrotron light sources and Free Electron Lasers (FELs) are valuable modern probes of matter with high temporal and spatial resolution. Laser Plasma Accelerators (LPAs), delivering GeV electron beams in few centimeters, are good candidates for future compact light sources. However the barriers set by the large energy spread, divergence and shot-to-shot fluctuations require a specific transport line, to shape the electron beam phase space for achieving ultrashort undulator synchrotron radiation suitable for users and even for achieving FEL amplification. Proof-of-principle LPA based undulator emission, with strong electron focusing or transport, does not yet exhibit the full specific radiation properties. We report on the generation of undulator radiation with an LPA beam based manipulation in a dedicated transport line with versatile properties. After evidencing the specific spatiospectral signature, we tune the resonant wavelength within $200-300 \mathrm{~nm}$ by modification of the electron beam energy and the undulator field. We achieve a wavelength stability of $2.6 \%$. We demonstrate that we can control the spatio-spectral purity and spectral brightness by reducing the energy range inside the chicane. We have also observed the second harmonic emission of the undulator.

Accelerator based light sources ${ }^{1}$ have experienced a remarkable increase of brilliance in the X-ray domain these last decades, transforming our understanding of the world using synchrotron light. Storage ring based facilities predominantly use insertion devices, commonly known as undulators, and benefit from the improvements of the electron beam parameters in particular the energy spread and emittance. They provide high brilliance X-ray radiation that addresses the $21^{s t}$ century societal challenges such as health, environment, energy, information technology and fundamental science. The radiation from electrons, wiggling in the undulator sinusoidal magnetic field, interfere constructively at the resonance wavelength and its harmonics, leading to a spectrum, consisting of sharp lines ${ }^{2-11}$ with a specific spatio-spectral distribution ("moon-shape" type pattern ${ }^{12-15}$ ). The generated radiation can acquire a high spectral purity and brightness, provided that the multi-electron contribution does not excessively affect on the interference process. The multi-electron degradation of the undulator spectral purity is mitigated thanks to improved electron beam performance, brought with the advent of diffraction limited storage rings ${ }^{16}$. Furthermore, the new laser revolution with the advent of $\mathrm{X}$-Ray $\mathrm{FEL}^{17}$, using relativistic electrons wiggling in an undulator as a gain medium in which light is amplified due to a stimulated Compton Backscattering process ${ }^{18}$,

${ }^{1}$ Synchrotron-SOLEIL, L'Orme des Merisiers, Saint-Aubin, Gif-sur-Yvette, 91192, France. ${ }^{2}$ Université Paris-Saclay, Paris, France. ${ }^{3}$ Univ. Lille, CNRS, UMR 8523 - PhLAM - Physique des Lasers Atomes et Molécules, 59000, Lille, France. ${ }^{4}$ LOA, ENSTA Paris, CNRS, Ecole Polytechnique, Institut Polytechnique de Paris, 828 Bd des Maréchaux, 91762, Palaiseau Cedex, France. ${ }^{5}$ Department of Physics of Complex Systems, Weizmann Institute of Science, Rehovot, 761001, Israel. ${ }^{6}$ NSLS-II, Brookhaven National Laboratory, 98 Rochester St, Upton, NY, 11973, USA. *email: amin. ghaith@synchrotron-soleil.fr 


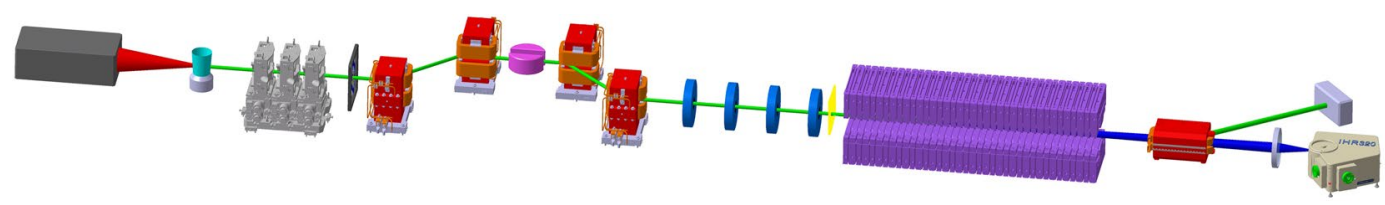

Figure 1. COXINEL Experimental set-up. Laser source (grey), gas jet (cyan), permanent magnet based quadrupoles (QUAPEVAs) (light grey), LANEX screen (black), electro-magnet dipoles (red) with an adjustable slit placed at the center (pink), electro-magnet quadrupoles (blue) with a $75 \mu \mathrm{m}$-thick Aluminum foil inserted at the center to remove plasma radiation and laser beam contamination (yellow), undulator (purple), dipole magnet (red) for electron beam dump (light purple), lens (grey) focusing the undulator radiation into a UV spectrometer (light grey).

brings a jump of several orders of magnitude in peak brightness. The high spectral purity and short pulses X-ray FELs open the path for deciphering un-explored ultra-fast phenomena ${ }^{19}$ with very high temporal resolution.

In view of miniaturizing accelerator based light sources, Laser Plasma Accelerator (LPA) ${ }^{20-25}$, with sub-PW class laser, serves as an attractive alternative to conventional Radio-Frequency (RF) acceleration. In an LPA, a high-power and ultra-short laser, focused into a gas target, drives a plasma wave that can trap and accelerate electrons from the ambient plasma ${ }^{26-28}$. LPA can deliver up to several $\mathrm{GeV}$ electron beams ${ }^{22,29}$ within a centimeter accelerating distance with low emittance ${ }^{30,31}$, few-femtosecond bunch length ${ }^{32}$ and high peak current ${ }^{33-35}$. While $\mathrm{RF}$ linacs deliver $1 \mathrm{nC}$ charge beams with microradian divergence and $\sim 0.01 \%$ energy spread, LPA still presents largely open challenges concerning the achievable energy spread ${ }^{36}$ at high charge operation, the initial divergence and shot-to-shot fluctuation. Indeed, the electron beam could be naturally deteriorated because of chromatic effects $^{37-39}$. Thus, special electron beam transport, with a well-designed phase-space manipulation from source to the undulator, is required for achieving narrow undulator radiation bandwidth and making LPA based FEL ${ }^{40-42}$ amplification possible ${ }^{43-48}$. For example, the emittance growth can be mitigated either using a plasma lens or high gradient quadrupoles. In addition, a magnetic chicane can be implemented ${ }^{43,45}$ to reduce the slice energy spread or the use of a transverse gradient undulator ${ }^{49}$ to compensate the effect of the energy spread.

Up to now, the LPA-based undulator radiation ${ }^{50-53}$ is still limited in terms of performance: large shot-to-shot spectral and intensity fluctuations, wide relative FWHM bandwidths $\left(7.5 \%^{50}, 16 \%{ }^{52}, 22 \%^{51}\right)$ resulting mainly from the large energy spread of the electron beam. Preliminary experimental results on undulator radiation have been observed after a dedicated manipulation transport line ${ }^{48}$. The full undulator features (wavelength tunability, spectral purity, stability...) suitable for taking advantage of this ultra-short undulator radiation for scientific applications have not been demonstrated so far. While the spectral purity of LPA based Compton sources ${ }^{54,55}$ allows for imaging applications ${ }^{56,57}$, the quality of the LPA based undulator radiation currently constitutes a challenge for being useable as a light source for scientific applications. As an associated prerequisite, reliable modeling of the undulator emission with a controlled electron beam is needed in view of synchrotron radiation and FEL application.

We report here that LPA electrons, in a phase-space manipulation transport line, can radiate in an undulator with properties approaching those achieved with conventional accelerators. The observed radiation presents the characteristic spatio-spectral distribution of the emitted light, exhibiting a "moon-shape" pattern as clear-cut evidence of the undulator radiation process. We then demonstrate that the electron beam control along the transport line enables us to achieve a proper reproducible wavelength tunabilty over $\sim 100 \mathrm{~nm}$ range by either electron beam energy or undulator magnetic field tuning. Finally, we show that we can achieve a spectral purity of $\frac{\Delta \lambda}{\lambda}=7.6 \%$ FWHM with a spectral peak brightness of $6 \times 10^{17} \mathrm{ph} / \mathrm{s} / \mathrm{mm}^{2} / \mathrm{mrad}^{2} / 0.1 \% \mathrm{BW}$.

\section{Results}

Experimental set-up and COXINEL transport manipulation line. Figure 1 presents the COXINEL experimental setup. A Titanium:Sapphire laser $(60 \mathrm{TW}, 30 \mathrm{fs})$ is focused on a supersonic gas jet $(99 \% \mathrm{He}$ and $1 \% \mathrm{~N}_{2}$ ). The LPA is operated in the robust ionization injection regime ${ }^{28}$ producing electron beams with a broad energy spectrum ending at $\sim 250 \mathrm{MeV}$, a typical charge density of $\sim 0.5 \mathrm{pC} / \mathrm{MeV}$ and a large divergence in the mrad range. The electrons are then transported and manipulated through the COXINEL beamline ${ }^{48,58}$. A triplet of strong tunable permanent magnet based quadrupoles (QUAPEVAs ${ }^{59}$, located immediately after the electron beam source, focuses the beam and mitigates the emittance growth. The beam is then longitudinally stretched by passing through a four-dipole magnetic chicane, where a variable width slit placed at the center selects a smaller energy range ${ }^{60-62}$. The optics ensures a proper energy selection via the slit (see Table 1), removal of the lateral "wings" of the transverse electron beam distribution and minimization of the beam size at the center of the undulator (see Methods). The Twiss parameters and emittance change significantly for different energies due to chromatic effects. Next, the electron beam goes into a second set of quadrupoles ensuring a focusing at the center of the undulator. The $18.16 \mathrm{~mm}$ period undulator can be adjusted between $4.55-30 \mathrm{~mm}$ gap attaining a peak field $B_{u}$ of $\sim 1.2 \mathrm{~T}$ at minimum gap ${ }^{63}$. The radiation is emitted in the Ultra-Violet (UV) for reference electron energies within 150-180 MeV (see Methods). Finally, the electron beam is dumped using a dipole magnet at the end of the transport line. The radiation is collected by a lens and focused at the entrance slit of a UV imaging spectrometer, enabling to map the radiation spatio-spectral distribution on a CCD camera (see Methods).

Observation of undulator spatio-spectral distribution. Figure 2(a) shows a single shot measurement of the spatio-spectral distribution of the UV light for an undulator gap of $5 \mathrm{~mm}$. The undulator radiation is 


\begin{tabular}{|l|l|l|l|l|l|l|l|l|}
\hline $\mathbf{E}$ & Slit & $\sigma_{\gamma}$ & $\sigma_{\boldsymbol{x}}$ & $\sigma_{{ }_{x}}^{\prime}$ & $\sigma_{z}$ & $\sigma_{z}^{\prime}$ & $\sigma_{\boldsymbol{l}}$ & $\mathbf{Q}$ \\
\hline $\mathbf{M e V}$ & $\mathbf{m m}$ & $\%$ & $\mu \mathbf{m}$ & $\mu \mathrm{rad}$ & $\mu \mathbf{m}$ & $\mu \mathrm{rad}$ & $\mu \mathbf{m}$ & $\mathbf{p C}$ \\
\hline 176 & 3 & 3.0 & 800 & 650 & 240 & 1100 & 140 & 2.5 \\
\hline 161 & 4 & 3.1 & 860 & 580 & 120 & 1450 & 135 & 5.6 \\
\hline 161 & 3 & 2.6 & 800 & 570 & 115 & 1390 & 115 & 4.8 \\
\hline 161 & 2 & 2.0 & 740 & 560 & 120 & 1290 & 90 & 3.7 \\
\hline 161 & 1 & 1.4 & 680 & 550 & 130 & 1060 & 60 & 2.1 \\
\hline
\end{tabular}

Table 1. Electron beam parameters at the undulator center. Electron energy spread $\sigma_{\gamma}$, transverse sizes (in horizontal $\sigma_{x}$, in vertical $\sigma_{z}$ ), divergences $\left(\sigma_{x}^{\prime}, \sigma_{z}^{\prime}\right)$, bunch length $\sigma_{l}$ and charge Q computed from the transport considering an initial total charge of $100 \mathrm{pC}$ for two different operating electron beam energies (see Methods).
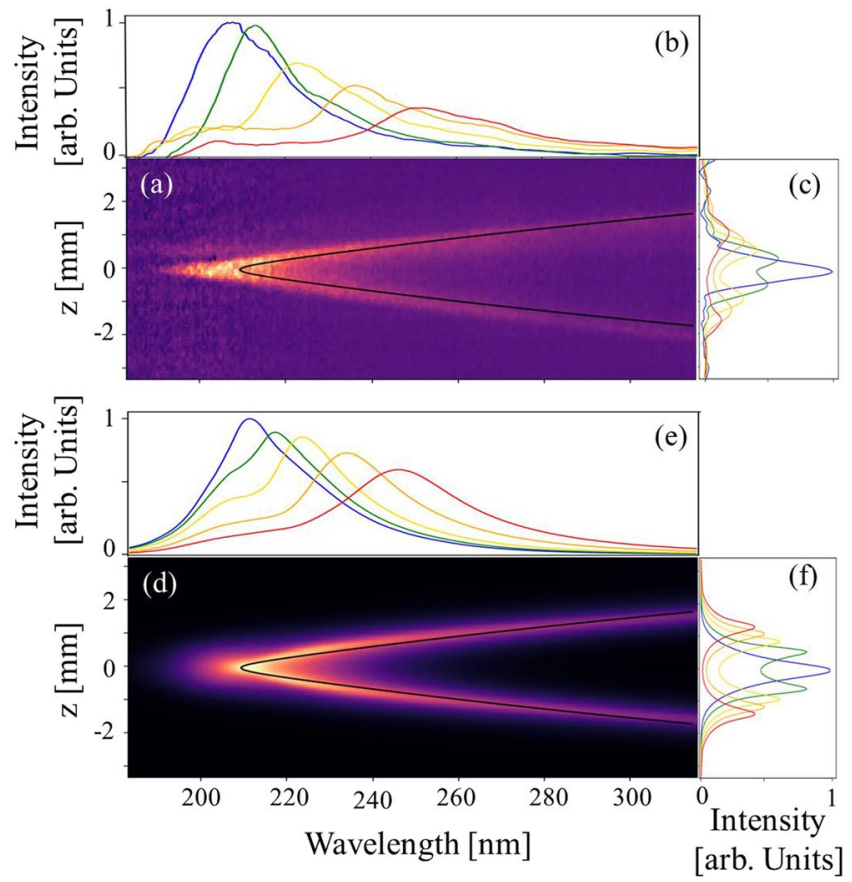

Figure 2. Measured and simulated undulator spatio-spectral distribution. (a) Single shot measurement for an electron beam energy of $176 \mathrm{MeV}$, a $5 \mathrm{~mm}$ undulator gap, $3 \mathrm{~mm}$ electron slit, $2.2 \mathrm{~mm}$ spectrometer slit width, and an applied calibration of the grating and CCD camera (See Methods). (d) Simulation using SRW with parameters of Table 1. (b) and (e) Undulator spectra for different vertical positions at $z=0$ (blue), $0.2 \mathrm{~mm}$ (green), $0.4 \mathrm{~mm}$ (yellow), $0.6 \mathrm{~mm}$ (orange), $0.8 \mathrm{~mm}$ (red). (c) and (f) Vertical radiation profiles with cuts at different wavelengths $\lambda=208 \mathrm{~nm}$ (blue), $228 \mathrm{~nm}$ (green), $248 \mathrm{~nm}$ (yellow), $268 \mathrm{~nm}$ (orange), $288 \mathrm{~nm}$ (red). Black curve: fit of the undulator resonance wavelength taking into account the chromatic aberrations of the lens (See Methods).

emitted at a resonant wavelength $\lambda=\lambda_{u}\left(1+K_{u}{ }^{2} / 2+\gamma^{2} \theta^{2}\right) / 2 n \gamma^{2}$, where $\lambda_{u}$ is the undulator period, $K_{u}$ the deflection parameter $\left(K_{u}=93.4 B_{u}[\mathrm{~T}] \lambda_{u}[\mathrm{~m}]\right), \gamma$ the Lorentz factor, $\theta$ the observation angle and $\mathrm{n}$ the harmonic number. The image exhibits a typical undulator "moon-shape" pattern resulting from the off-axis emission ${ }^{12-14}$. The off-axis radiation at vertical position $(|z|>0)$ is red-shifted due to the $\gamma^{2} \theta_{z}^{2}$ term in the resonance wavelength relationship (for example $232 \mathrm{~nm}$ for $|z|=0.6 \mathrm{~mm}$ compared to $210 \mathrm{~nm}$ on-axis) and has lower intensity. The effect is more pronounced for larger angles of observation. The measurement is compared to simulation (see Fig. 2(d)) performed with the SRW code ${ }^{6}$. The beam parameters at undulator entrance are computed using the measured initial electron beam parameters transported along the beamline (see Methods). The far-field undulator radiation is computed separately for each energy slice and the resulting intensities are summed. The lens imaging ratio is applied including its chromatic corrections. The simulation and measurement show similar "moon-shape" patterns. The triangular shape, slightly deviating from the usual parabolic behaviour due to the $\gamma^{2} \theta_{z}^{2}$, results from the chromatic effects of the lens (see Methods).

Figure 2(b,e) show the vertical angular dependance of the spectra with the image cuts at various $z$. The on-axis $(z=0)$ spectrum is peaked at the resonance wavelength of $208 \mathrm{~nm}$ with a $13.1 \%$ relative FWHM bandwidth, larger than the natural homogeneous linewidth $\left((\Delta \lambda / \lambda)_{\text {hom }}=1 / \mathrm{nN}\right.$ with $\mathrm{N}$ the number of undulator periods $(0.84 \%$ for $\mathrm{N}=107)$ ) by one order of magnitude. This so-called inhomogeneous broadening results from the multi-electron contribution, and thus from the electron beam parameters. The relative energy spread $\sigma_{\gamma}$ symmetrically widens 

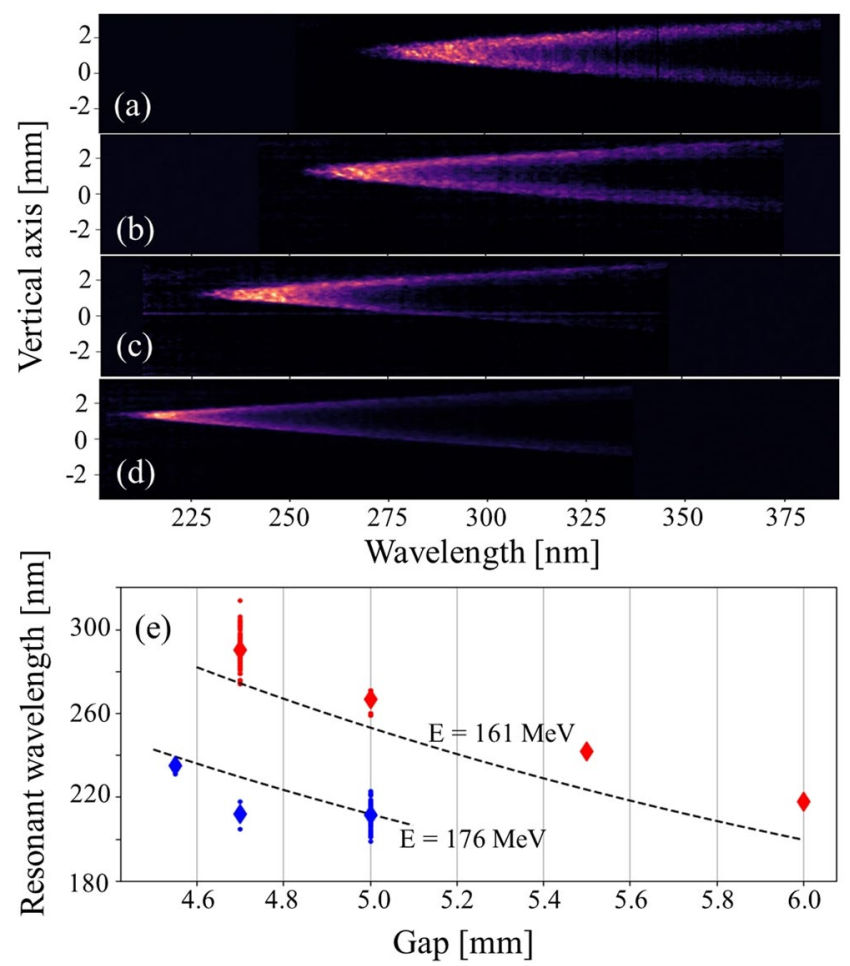

Figure 3. Wavelength tunability by undulator gap and energy change. Single shot spatio-spectra distribution measured for $161 \mathrm{MeV}$ beam energy at different gaps: (a) $4.7 \mathrm{~mm}$, (b) $5 \mathrm{~mm}$, (c) $5.5 \mathrm{~mm}$, (d) $6 \mathrm{~mm}$, with an electron slit of $1 \mathrm{~mm}$ and a spectrometer slit of $2.2 \mathrm{~mm}$. (e) Measured and theoretical (dashed) resonant wavelength versus undulator gap: $161 \mathrm{MeV}$ (red) and $176 \mathrm{MeV}$ (blue).

the spectral line, as $(\Delta \sigma / \gamma)_{\sigma \gamma} \simeq 2 \sigma_{\gamma}\left(\sim 14 \%\right.$ FWHM for $\sigma_{\gamma}$ of $3 \%$ RMS $)$. The emittance broadens the spectral flux distribution integrated over $\mathrm{z}$ mainly on the "red" side. At $\mathrm{z}=0$, only the horizontal divergence $\left(\frac{\Delta \lambda}{\lambda}\right)_{\sigma^{\prime}} \simeq \frac{\gamma^{2} \sigma_{x}^{\prime 2}}{1+K_{u}^{2} / 2}$ ( $\sim 8 \%$ for 0.65 mrad RMS) brings a non-negligible contribution. The vertical radiation profiles for increasing wavelengths, shown in Fig. 2(c,f), exhibit first a Gaussian type distribution $(\lambda=208 \mathrm{~nm})$ followed by a hole dip in the center $(\lambda=228 \mathrm{~nm})$ and a donut shape $(\lambda=268 \mathrm{~nm}$ and above), as typically observed for undulator radiation $^{9,15}$.

Undulator radiation tunability. The undulator radiation tunability, one of the major undulator properties, is then explored. The radiated wavelength is independently varied either by changing the undulator gap, or through focusing a different energy by quadrupole gradients and chicane strength adjustments ${ }^{48}$. Such an undulator wavelength control corresponds to what is currently achieved with conventional accelerators. Figure 3(a-d) show the measured spatio-spectral distribution for different undulator gaps. The smaller the gap, the larger the resonant wavelength. Figure 3(e) displays the evolution of the measured resonant wavelength versus gap for two different energy settings. The behaviour shows good agreement with theoretical curves calculated using the measured magnetic field gap dependence (see Methods). The discrepancy between theory and experiment in the 161 $\mathrm{MeV}$ case could be a result of the laser degradation (orbit change leading to a variation in electron beam energy and misalignment in the undulator); a vertical misalignment of the electron beam with respect to the undulator axis (a $0.5 \mathrm{~mm}$ deviation leads to $1.5 \%$ field variation); the uncertainty of the undulator peak field at $4.7 \mathrm{~mm}$ gap extrapolation (see Methods) (a 5\% field deviation corresponds to a $\sim 7 \%$ resonant wavelength variation). The tunability is typically achieved between $210 \mathrm{~nm}$ and $300 \mathrm{~nm}$. The photon flux increases for smaller gap, but the spectral brightness is maximum at a gap of $4.5 \mathrm{~mm}(\mathrm{~K}=2)$.

Undulator radiation wavelength stability. The stability of the undulator resonant wavelength was investigated. The electron beam manipulation transport line controls the proper focusing of the electron beam energy of interest in the undulator, and the slit selects a given electron beam energy range. Figure 4 displays the undulator resonant wavelength evolution during 3 hours. The wavelength is centered, on average, at $290 \mathrm{~nm}$, with a RMS value of $7.8 \mathrm{~nm}$ over 60 shots, corresponding to a $2.6 \%$ stability.

Undulator radiation bandwidth control. After having evidenced the typical features of the measured undulator radiation, the control of the spectral bandwidth is then examined. Figure 5(a-d) show the undulator spatio-spectral patterns, with the corresponding appended on-axis spectra, measured while shaping the beam parameters. As the slit is closed in the magnetic chicane, the beam energy spread, size and divergence in the undulator are reduced (see Table 1) and, accordingly, the corresponding measured moon-shape thickness decreases. Without the slit, the energy range is broad, the resonant wavelength spans a large range and the moon-shape 


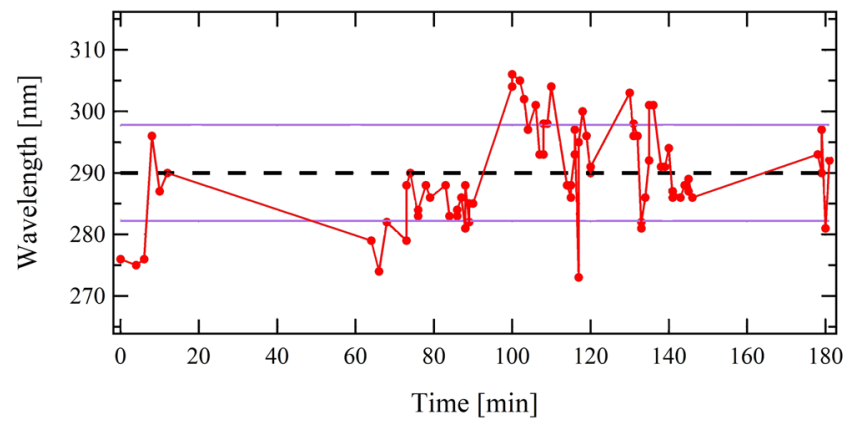

Figure 4. Resonant wavelength stability. Undulator resonant wavelength measured during 60 successive shots over 3 hours for an undulator gap of $4.7 \mathrm{~mm}$ and different electron slit widths. Average value (dashed), standard deviation (purple).
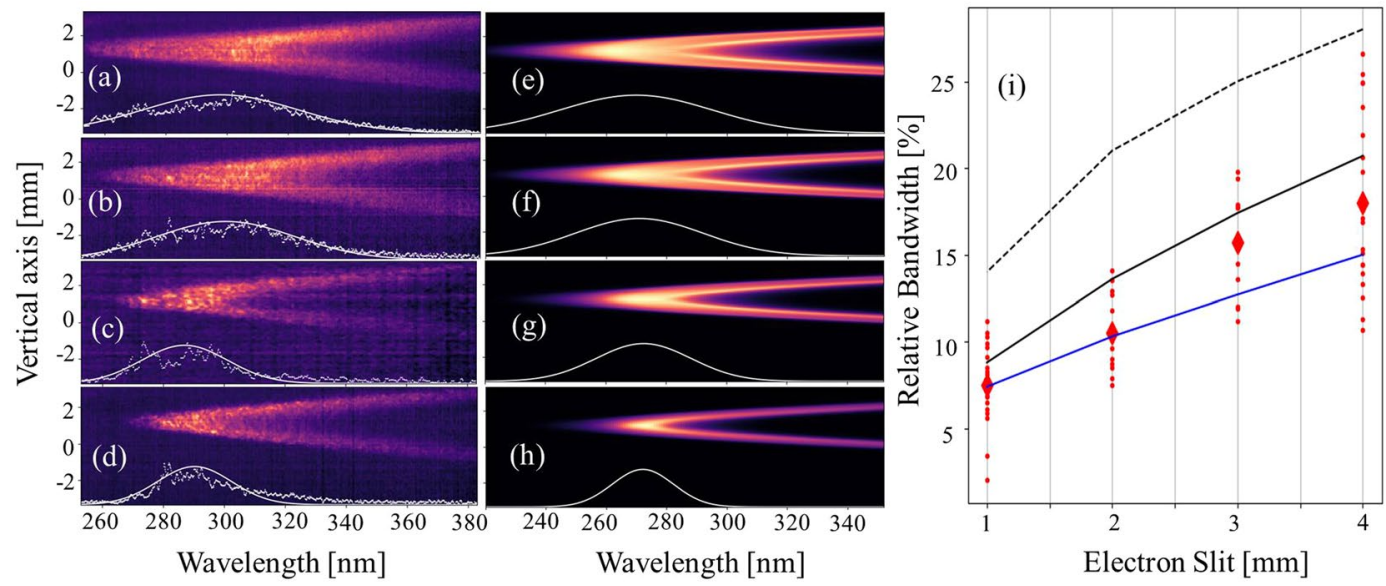

Figure 5. Undulator spatio-spectral distribution dependance on the electron beam energy selection. Single shot measured spatio-spectral distributions for a $4.7 \mathrm{~mm}$ undulator gap (with caibration) while varying the electron slit width: 4 (a), 3 (b), 2 (c) and $1 \mathrm{~mm}$ (d) with a $2.2 \mathrm{~mm}$ spectrometer entrance slit. Simulated spectra using SRW for a magnetic field of $1.17 \mathrm{~T}$, with beam parameters taken from the simulations of the corresponding electron beam distribution transported along the line (see Table 1 for $161 \mathrm{MeV}$ ) for slit widths of 4 (e), 3 (f), 2 (g) and $1 \mathrm{~mm}$ (h) with their corresponding on-axis spectra (white curves). (i) Measured (red), analytically estimate of energy spread contribution (blue), analytically estimate of all the contributions (dashed) and simulated (line) FWHM relative bandwidth of the on-axis spectra.

patterns from different energy electrons are overlapped, resulting in a strong smearing of the spatio-spectral distribution. Figure 5(i) shows the measured on-axis radiation bandwidth versus chicane slit widths. For the $1 \mathrm{~mm}$ slit width, the average relative bandwidth is found to be $7.6 \%$ with a standard deviation of $2 \%$ over 21 shots and the lowest undulator spectral bandwidth achieved is $2 \%$.

These measurements can be first interpreted with an analytic approach as plotted in Fig. 5(i) using the average parameters in Table 1 with two extreme cases. In the first limiting case (blue line), where only the inhomogeneous broadening of the energy spread and horizontal divergence is considered, the observed trend of the linewidth increase is well reproduced, while the analytic estimate stands below the measured data for larger slit width since some of the contributions are neglected. The second limiting case (black line), where all contributions (emittance and energy spread) to the inhomogeneous broadening are taken into account (quadratic sum), shows a homothetic evolution same to the experimental one: the slope is mainly determined by the energy spread, with an additional linewidth widening due to the beam size and divergence increase for larger slit width. This second limiting case is situated above the measurements, since the assumption of considering separately the emittance and energy spread contributions becomes less valid for large energy spread values. The two extreme analytic cases surround the measurements. A more precise analysis of the measurements can be performed using a comparison with the simulations from SRW with the electron beam distribution at the undulator center for different electron slit widths (see Methods), as shown in Fig. 5(e-h). A quantitative agreement is achieved between measurements and simulations as shown in Fig. 5(i). The simulated bandwidths (black solid line) produce an improved agreement to the measured ones (compared to the analytic estimates), due to a proper multi-electron treatment.

The undulator spatio-spectral pattern has been successfully controlled via selecting a specific energy range of the electron beam in the transport line while transmitting the charge of the electrons at the energy of interest, 

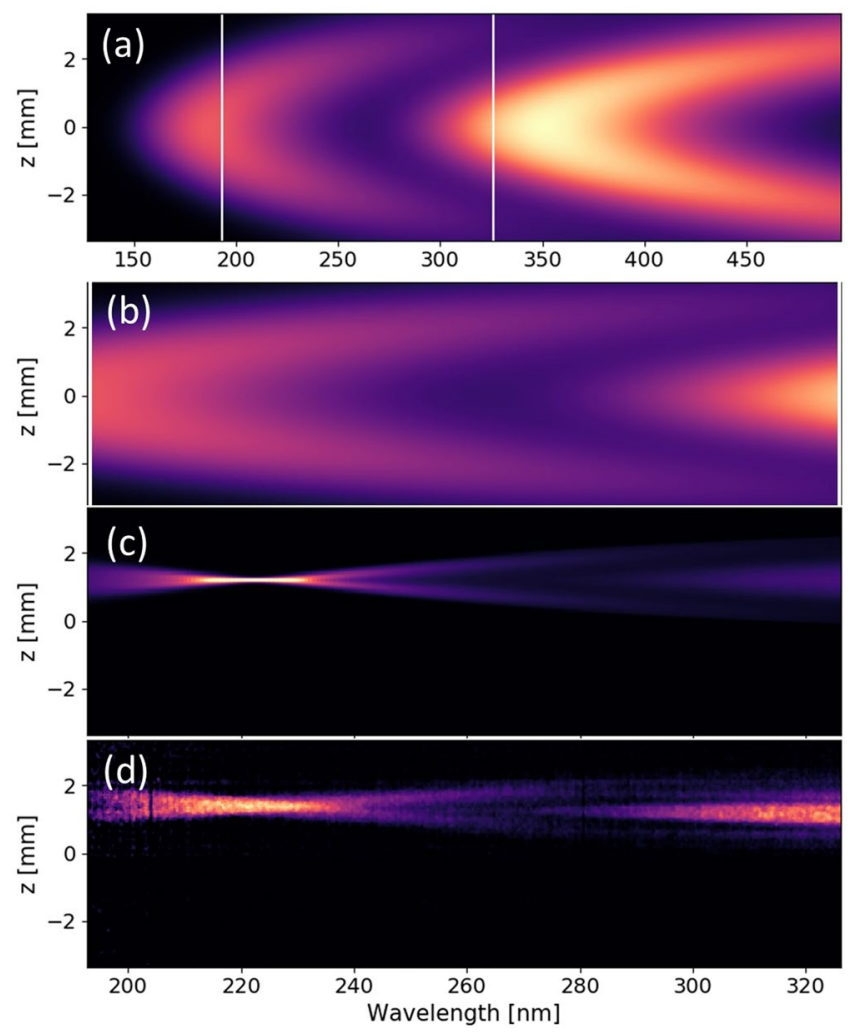

Figure 6. Observation of the second undulator harmonic. Undulator radiation spatio-spectral distribution at a gap of $4.7 \mathrm{~mm}$, electron slit opened at $4 \mathrm{~mm}$ and spectrometer slit at $2.2 \mathrm{~mm}$. (a) Simulation showing the first and second harmonic, (b) zoom of (a), (c) simulation including chromatic effects of the lens on (b), (d) calibrated measurement.

and cleaning the ones for lower and higher energies. As a result, the spectral purity of the undulator radiation has been improved. The coherence length of the radiation is increased by a factor of 2.3 from the $4 \mathrm{~mm}$ electron slit case $(4.7 \mu \mathrm{m})$ to the $1 \mathrm{~mm}(10.8 \mu \mathrm{m})$ one. Controlling directly the spectral bandwidth with the electron beam energy spread, instead of using a photon monochromator, enables us to preserve the radiation wavefront and avoid intensity reduction.

Undulator spectral brightness. The behavior of the photon angular flux and spectral brightness, i.e. the photon flux over transverse and longitudinal phase space area, is then analyzed versus the electron energy control. The calculations (see Methods) are done using the LPA average beam parameters deduced from the transport simulations of Table 1 (see Methods). The peak spectral brightness is calculated to be $\sim 2 \times 10^{17} \mathrm{ph} / \mathrm{s} / \mathrm{mm}^{2} /$ $\mathrm{mrad}^{2} / 0.1 \%$ BW and $\sim 6 \times 10^{17} \mathrm{ph} / \mathrm{s} / \mathrm{mm}^{2} / \mathrm{mrad}^{2} / 0.1 \%$ BW using average bandwidths for an electron slit width of $4 \mathrm{~mm}$ and $1 \mathrm{~mm}$, respectively. Indeed, for smaller electron slit widths, the total beam charge is reduced, whereas the charge for the energy of interest remains practically the same. Spectral bandwidth is narrowed due to energy spread and emittance terms, where chromatic growth is mitigated by the energy control. In consequence, the peak photon beam brightness increases. Taking the $10^{\text {th }}$ percentile of the bandwidth measured in the $1 \mathrm{~mm}$ slit case (3.4\%), the maximum calculated spectral brightness is found to be $\sim 1 \times 10^{18} \mathrm{ph} / \mathrm{s} / \mathrm{mm}^{2} / \mathrm{mrad}^{2} / 0.1 \%$ BW.

Observation of second harmonic. One of the characteristic features of planar undulators is the high intensity emission on the harmonics, provided the deflection parameter is not too small. Considering the limited spectral range of the used spectrometer, the emission on the second harmonic is explored. Figure 6(a,b) present a simulation using the average beam parameters for the $4 \mathrm{~mm}$ slit case (see Table 1). The second harmonic is visible on-axis $(175 \mathrm{~nm})$. The single-electron undulator radiation at even harmonics is known to be suppressed in the direction along the electron motion axis, because the emitted electric field at these harmonics is anti-symmetric with respect to transverse position/angle in the plane containing the electron trajector $y^{64}$ and a significant photon flux is emitted off-axis at even harmonics. The observed on-axis radiation at $175 \mathrm{~nm}$ results from the contribution of the finite electron beam emittance (angular divergence at the observation in the far field and transverse size at the observation in a plane of source imaging). Figure 6(d) shows a measurement of undulator radiation, where the moon shape of the second harmonic is twisted (closed onto itself) due to chromatic effects of the lens, forming a "ribbon" type pattern. The maximum intensity is observed at $225 \mathrm{~nm}$, where the focal length of this particular wavelength is equal to the distance between the lens and the spectrometer slit (see Methods). Figure 6(c) displays the simulated undulator radiation of (b) after introducing the chromatic effects. A good agreement is found with the measurement. 


\section{Discussion}

We have examined the characteristics of LPA generated undulator radiation after a manipulation beam line. We have shown that it can exhibit the distinguished properties currently observed on conventional accelerator light sources, with the particular spatio-spectral dependance leading to a moon-shape pattern. The proper handling of the energy of interest along the COXINEL line ensures a stability of the resonant wavelength within $2.4 \%$. A $\sim 100 \mathrm{~nm}$ tunability of LPA based undulator radiation has been achieved by varying the undulator gap and electron beam energy. Furthermore, the control of the undulator radiation has been accomplished via chicane and slit combination, resulting in a small radiation bandwidth down to 7.6\% FWHM and achieving a high peak photon beam spectral brightness up to $6 \times 10^{17} \mathrm{ph} / \mathrm{s} / \mathrm{mm}^{2} / \mathrm{mrad}^{2} / 0.1 \%$ BW. The satisfactory comparison between measurements and simulations using electron beam longitudinal and transverse distributions make us confident in future predictions of undulator radiation. The achieved agreement demonstrates a new capability in handling the LPA electron beam and transporting it to the undulator for a high photon beam brilliance.

Although the laser plasma acceleration set in a robust configuration (ionization injection) usually delivers electron beams with large energy spread and divergence typically above $1 \mathrm{mrad}$ RMS, the control of the undulator spontaneous emission after a dedicated transport manipulation line constitutes an acquired maturity and a major step forward. One can extrapolate these results to a $1 \mathrm{GeV}$ beam with initial typical parameters achieved ${ }^{21,22,29,34,35}$ $\left(\sigma_{z}^{\prime}=2 \mathrm{mrad}, \sigma_{\gamma}=2.5 \%\right)$ with $50 \mathrm{pC}$ charge, and re-optimizing the COXINEL line to transport these high energy beams with new magnetic element settings. For a $1 \mathrm{~mm}$ electron slit, the peak spectral brightness from the present undulator could reach $\sim 4 \times 10^{21} \mathrm{ph} / \mathrm{s} / \mathrm{mm}^{2} / \mathrm{mrad}^{2} / 0.1 \%$ BW at a resonant wavelength of $6.5 \mathrm{~nm}$. If one would replace the undulator by a LUNEX 5 type ( $3 \mathrm{~m}$ long cryogenic undulator of period $15 \mathrm{~mm}$ and $K_{u} \sim 2^{65}$ ), the peak brilliance is calculated to be $\sim 1 \times 10^{22} \mathrm{ph} / \mathrm{s} / \mathrm{mm}^{2} / \mathrm{mrad}^{2} / 0.1 \% \mathrm{BW}$ at a resonant wavelength of $7 \mathrm{~nm}$, as compared to those achieved using high harmonic in gas $\left(10^{19}-10^{23} \mathrm{ph} / \mathrm{s} / \mathrm{mm}^{2} / \mathrm{mrad}^{2} / 0.1 \% \mathrm{BW}\right)^{66}$ and synchrotron radiation $\left(10^{19}-10^{25} \mathrm{ph} / \mathrm{s} / \mathrm{mm}^{2} / \mathrm{mrad}^{2} / 0.1 \% \mathrm{BW}\right)^{67}$.

These results pave the way for providing reliable ultra-short LPA based X-ray undulator radiation for serving the scientific user community in a configuration designed for short wavelength operation, as planned on ELI (Extreme Light Infrastructure $)^{68}$.

\section{Methods}

Laser plasma accelerator. The laser-wakefield accelerator is driven by a Titanium:Sapphire laser system at Laboratoire d'Optique Appliquée, which delivers $30 \mathrm{fs}$ (FWHM) pulses of $1.5 \mathrm{~J}$ energy at a central wavelength of $800 \mathrm{~nm}$ with a repetition rate of $1 \mathrm{~Hz}$. The laser beam is focused by an off-axis parabola into a gas mixture composed of $99 \% \mathrm{He}$ and $1 \% \mathrm{~N}_{2}$. A retractable electron spectrometer, placed after the supersonic gas jet with $3 \mathrm{~mm}$ exit diameter, measures the electron beam divergence along the vertical axis (perpendicular to the laser polarisation axis), initial energy spectrum and the charge density. A first electron beam imager located $64 \mathrm{~cm}$ away from the electron source enables to measure the beam horizontal and vertical divergences as well as electron beam pointing stability.

Two experimental settings have been used for the photon measurements, with a beam transport at $161 \mathrm{MeV}$ (see Fig. 7(a)) and at $176 \mathrm{MeV}$ energy (see Fig. 7(b)). In the $176 \mathrm{MeV}$ case, the average vertical divergence measured on the spectrometer presents an average of $3.2 \mathrm{mrad}$ RMS (Standard Deviation (SD) of $0.5 \mathrm{mrad}$ ) over 20 shots over the whole distribution, and drops to $2.1 \mathrm{mrad}$ RMS ( $\mathrm{SD}$ of $0.3 \mathrm{mrad}$ over 20 shots) for the $176 \pm 5 \mathrm{MeV}$ slice, with an average charge of $3.1 \pm 1 \mathrm{pC}$ within this slice. The electron beam pointing stability, measured on the first screen, is $1.2 \mathrm{mrad}$ over 20 shots. In the $161 \mathrm{MeV}$ case, the average vertical divergence measured on the spectrometer presents an average of $1.95 \mathrm{mrad}$ RMS (SD of $0.3 \mathrm{mrad}$ ) over 40 shots over the whole distribution, and of $1.85 \mathrm{mrad}$ RMS (SD of $0.2 \mathrm{mrad}$ over 40 shots) for the $\pm 5 \mathrm{MeV}$ slice, with an average charge of $6.0 \pm 1$ $\mathrm{pC}$ within this slice. The electron beam pointing stability, measured on the first screen, is $1.2 \mathrm{mrad}$ over 5 shots.

The electron beam transport in the COXINEL line. The COXINEL line uses permanent magnet based quadrupoles with tunable high gradient (QUAPEVAs) ${ }^{59,69-71}$, providing a tunability of $\sim 45 \%$ within $10 \mu \mathrm{m} \mathrm{mag-}$ netic center change. The relative gradient precision of $6 \times 10^{-4}$ results from the stretched wire ${ }^{72}$ measurements. The QUAPEVAs are mounted on translation stages, that are used for the beam pointing alignment procedure ${ }^{48}$. The magnetic chicane consists of four $\times 25 \mathrm{~mm}$ yoke gap water-cooled dipoles creating a $0.55 \mathrm{~T}$ magnetic field, measured with relative error of $1.8 \%$ for $150 \mathrm{~A}$. Inside the chicane is inserted a removable slit of variable width $l$ (up to $4 \mathrm{~mm}$ ) at a horizontal position of $32 \pm 0.1 \mathrm{~mm}$ corresponding to the $176 \mathrm{MeV}$ energy. The slit, machined in a stainless steel $304 \mathrm{~L}$ cylinder of $35 \mathrm{~mm}$ diameter and mounted on an under-vacuum plunger can be rotated with a MagicDrive (MD40SSES1X000Z) coupled with a stepper motor. The slit width calibration is given by: $l[\mathrm{~mm}]=-0.5256 \times\left(\theta+\theta_{\text {offset }}\right)+4.0034$, with $\theta$ the rotation angle and $\theta_{\text {offset }}$ the offset angle, using the electron beam transmission along the line for the $\theta_{\text {offset }}$ estimation $\left(-1.6^{\circ}\right.$ for $176 \mathrm{MeV}$ and $0^{\circ}$ for $\left.161 \mathrm{MeV}\right)$ and the CATIA model for the slope evaluation. Two Turbo Integrating Current Transformers (T-ICT from Bergoz, with $10 \mathrm{fC}$ noise) after the electron generation chamber and at the exit of the undulator, measure the electron charge, giving consistant measurements with the charge density measured on the electron spectrometer.

Electron beam transport is tuned with BETA code ${ }^{73}$ up to the second order, with multi-particle tracking code for high order non-linear effects and collective effects such as Coherent Synchrotron Radiation ${ }^{74}$. Hard edge models are used for the magnets and apertures of the vacuum chamber along the line are included. For the simulations, the electron beam distribution (charge, divergence) is deduced from an average of the measured ones prior to and after the data measurements. The optics, plotted in Fig. 7(c,d), selects a smaller range of energies and minimizes the beam size at the center of the undulator thanks to the slit. The Twiss parameters at the undulator center are given in Fig. 7(e,f). The energy spread is reduced for smaller slit width (see Fig. 7(g,h) and Table 1), whereas the lateral wings of the transverse electron beam distribution are removed (see Fig. $7(\mathrm{i}, \mathrm{j})$ ). 

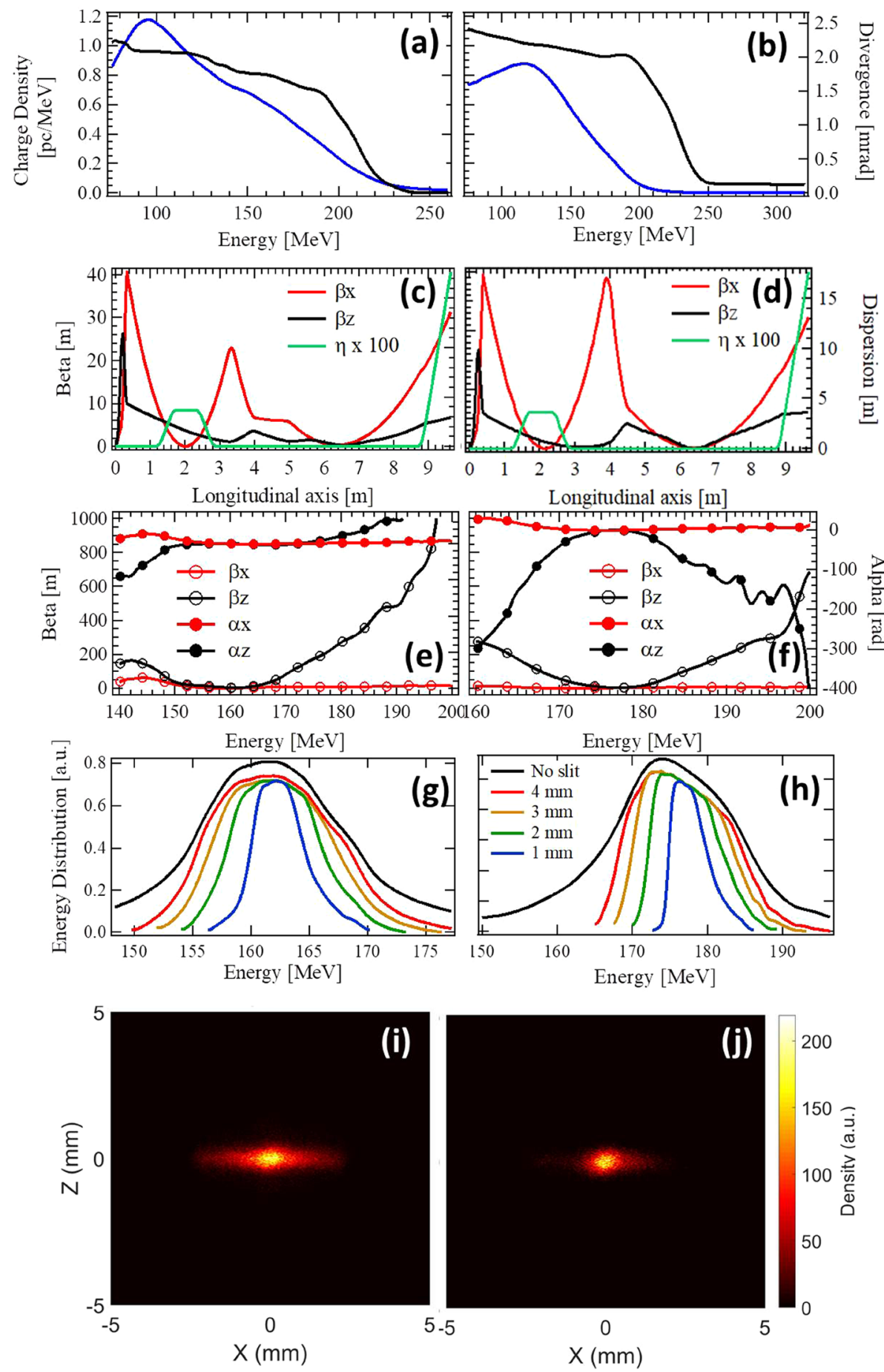

Figure 7. Measured electron beam charge distribution (blue), vertical divergence (black) for 161 (a) and 176 (b) $\mathrm{MeV}$, with $\sigma_{x}^{\prime} / \sigma_{z}^{\prime}=1.56$. Twiss parameters (betatron and dispersion) evolution simulation for a monoenergetic electron beam of 161 (c) and 176 (d) $\mathrm{MeV}$ along the transport line. Twiss parameters (beta and alpha) at the undulator center versus energy slice for the 161 (e) and 176 (f) $\mathrm{MeV}$ cases. Energy distribution at the undulator center for different slit widths: no slit (red), 3.2 (orange), 2.2 (green), 1 (blue) mm width for the 161 (g) and 176 (h) $\mathrm{MeV}$ cases. Electron beam transverse distribution at undulator center for the $161 \mathrm{MeV}$ : (i) no slit and (j) $1 \mathrm{~mm}$ slit. Parameters for the transport calculations: $1 \mathrm{~mm}$.mrad initial emittance, $1 \mu \mathrm{m}$ longitudinal size, $10^{6}$ macroparticles, $4.3 \mathrm{~mm}$ chicane strength. In the 176 (resp. 161) MeV case, QUAPEVA 1 of $40.7 \mathrm{~mm}$ magnetic length: +104.1 $\mathrm{T} \mathrm{m}^{-1}$ (resp. 113.5), QUAPEVA 2 of $44.7 \mathrm{~mm}$ magnetic length: $-103.1 \mathrm{~T} \mathrm{~m}^{-1}$ (resp. - 111.3), QUAPEVA 3 of $26 \mathrm{~mm}$ magnetic length: $+96.4 \mathrm{~T} \mathrm{~m}^{-1}$ (resp. 103.4). QUAPEVA skew contribution (ratio of skew gradient over normal gradient) of $+1.5 \times 10^{-3}$ (QUAPEVA 1$),-0.3 \times 10^{-3}$ (QUAPEVA 2$),-0.7 \times 10^{-3}$ (QUAPEVA 3) with a field variation of $2 \%$ at $4-\mathrm{mm}$ radius due to a dodecapole component for the three QUAPEVA. Electromagnetic quadrupole gradients at 176 (reps. 161) $\mathrm{MeV}:-0.01,4.7,-4.4,+0.29 \mathrm{~T} \mathrm{~m}^{-1}(4.15$, $-3.45,-0.13,+1.7 \mathrm{~T} \mathrm{~m}^{-1}$ ) for QEM $1,2,3,4$. 
The initial electron beam distribution is not measured simultaneously with the undulator pattern since it would intercept the beam, so it becomes a source of error in the modeling since the distribution can vary from shot-to-shot. The sensitivity to the parameters along the electron beam transport and the undulator radiation emission is considered. Simulations with emittance of $0.2 \pi \mathrm{mm}$. mrad lead to a $4 \%$ change of the radiation spectral bandwidth in the $4 \mathrm{~mm}$ electron slit case. A $0.5 \mathrm{mrad}$ RMS divergence difference in the initial distribution results in $10 \%(15 \%, 7 \%)$ change in energy spread (respectively for transverse beam sizes and divergences), resulting in a change of $1.3 \%$ of the relative bandwidth for the $4 \mathrm{~mm}$ electron slit. These assumptions do not significantly affect the result and are reduced for smaller electron slit width.

The undulator. The photon source is a $2 \mathrm{~m}$ long hybrid cryo-ready undulator of 107 periods $(18.16 \mathrm{~mm})$ operating at room temperature with adjustable gap ${ }^{63}$ built at Synchrotron SOLEIL. It consists of $\operatorname{Pr}_{2} \mathrm{Fe}_{14} B$ magnets (remanence field of $1.32 \mathrm{~T}$ and coercivity of $1930 \mathrm{kA} / \mathrm{m}$ at room temperature) and Vanadium Permendur poles (field saturation at $2.35 \mathrm{~T}$ ). The magnetic field is measured using a Hall probe with a precision of 0.5 Gauss and computed using RADIA ${ }^{75}$. At minimum gap $g$ of $5 \mathrm{~mm}$, the corresponding peak field, $B_{\text {peak }}$, is $1.1 \mathrm{~T}$. In the experiment, the undulator was closed to a gap of $4.55 \mathrm{~mm}$ due to the weak spectrometer response at wavelengths below $200 \mathrm{~nm}$. Thus, the peak field at gaps smaller than $5 \mathrm{~mm}$ have been estimated by extrapolating from the measured field. $B_{\text {peak }}$ versus $g$ measured is fitted with $B_{\text {peak }}=a \cdot \exp \left[b \frac{g}{\lambda_{u}}+c\left(\frac{g}{\lambda_{u}}\right)^{2}\right]$ for gaps between $5 \mathrm{~mm}$ and $10 \mathrm{~mm}$, where $(a, b, c)=(3.37,-4.34,1.12)$.

Lens. The lens used (eSource Optics CF5025LCX) is of spherical shape made of Calcium Flouride $\left(\mathrm{CaF}_{2}\right)$ and placed at a distance of $2.5 \mathrm{~m}$ from the undulator exit. The focal length $f$ depends on its refractive index $n$ as $\frac{1}{f(\lambda)}=\frac{n(\lambda)-1}{R}$ with $R=108.5 \mathrm{~mm}$ the radius of curvature of the lens. The chromatic dispersion is developed using the Sellmeier coefficients as $n(\lambda)^{2}-1=\frac{B_{1} \lambda^{2}}{\lambda^{2}-C_{1}^{2}}+\frac{B_{2} \lambda^{2}}{\lambda^{2}-C_{2}^{2}}+\frac{B_{3} \lambda^{2}}{\lambda^{2}-C_{3}^{2}}$ and $B_{1,2,3}, C_{1,2,3}$ with values from ${ }^{76}$. $B_{1}=0.5675888, B_{2}=0.4710914, B_{3}=3.8484723, C_{1}=0.050263605 \mu \mathrm{m}, C_{2}=0.1003909 \mu \mathrm{m}, C_{3}=34.649040 \mu \mathrm{m}$

Photon spectrometer. The undulator radiation is focused with the lens onto the entrance slit of the spectrometer (Horiba IHR320) located at distance $d$ from the slit, that can be varied from $80 \mu \mathrm{m}$ up to $2.2 \mathrm{~mm}$. The spectrometer, equipped with a 600 groove $/ \mathrm{mm}$ grating with an average reflectivity of $\sim 45 \%(200-300 \mathrm{~nm})$ and a linear dispersion of $4.917 \mathrm{~nm} / \mathrm{mm}$, enables to map the radiation vertical spatio-spectral distribution with a magnification factor from entrance slit to CCD camera $G=1.1$ on a UV-sensitive CCD (Horiba instruments SYNAPSE - 354308) consisting of $1024 \times 256$ pixels with a size of $26 \mu \mathrm{m}$ covering an area of $26.6 \mathrm{~mm} \times 6.7 \mathrm{~mm}$ $(133 \mathrm{~nm} \times 6.7 \mathrm{~mm})$ and a quantum efficiency of $\sim 60 \%$ for wavelengths between $200 \mathrm{~nm}$ and $300 \mathrm{~nm}$. The resolution variation versus slit width, measured with two lasers (green and red) is fitted with $c s_{w}+d$, where $s_{w}$ is the slit width in $\mathrm{mm}, c=5.34 \mathrm{~nm} / \mathrm{mm}$ and $d=0.031 \mathrm{~nm}$. For the data analysis, the background noise was removed, a median filter applied and convoluted with the spectrometer response.

Modeling of the undulator radiation. The electron beam parameters deduced from the measured distribution and transported along the line are used for the undulator radiation modeling using SRW code ${ }^{6}$ in the far-field region. A so-called slicing method is used, where radiation of each electron energy slice is computed separately with its corresponding parameters (divergence and size), and then all the spectra are added up taking into account the slice energy distributions. Ray optics is then applied to the computed undulator radiation, assuming that the lens position is in the far-field. The undulator radiation emitted with an angle $\theta_{z 0}$ impinges the lens at a height $z_{1}=D_{\text {lens }} \theta_{z 0}+h$, with $h$ the offset between the undulator radiation optical axis and $D_{\text {lens }}$ the distance between the undulator center and the lens. Using the matrix formalism and the chromatic aberration effect, the wavelength-dependent conversion from $\theta_{z 0}$ to spectrometer CCD vertical $z_{C C D}$ :

$$
z_{C C D}=G \times\left[z_{1}(1-d / f(\lambda))+d \theta_{z_{0}}\right]+H
$$

with $H$ the offset between the camera center and the lens optical axis. The measured moon-shape pattern of Fig. 2(a) is fitted by using the resonant wavelength relationship and Eq. 1 to deduce d, h, $\mathrm{H}$ and the electron beam energy.

Spectral brightness. The spectral peak brightness is estimated using expression given in ${ }^{77}$, with analytical calculation of the flux (benchmarked with SRW code), the phase space transverse dimension, peak current deduced from Table 1 and measured undulator relative bandwidth.

\section{Data availability}

The data that support the findings of this study are available from the corresponding authors upon reasonable request.

Received: 17 April 2019; Accepted: 22 October 2019;

Published online: 13 December 2019

\section{References}

1. Couprie, M.-E. \& Filhol, J.-M. X radiation sources based on accelerators. Comptes Rendus Physique 9, 487-506 (2008).

2. Medvedev, A. \& Nikitin, M. Undulator radiation. Russian Physics Journal 17, 1452-1453 (1974).

3. Hofmann, A. Quasi-monochromatic synchrotron radiation from undulators. Nuclear Instruments and Methods 152, 17-21 (1978).

4. Coisson, R. Angular-spectral distribution and polarization of synchrotron radiation from a "short" magnet. Physical Review A 20, 524 (1979). 
5. Coisson, R. Spatial coherence of synchrotron radiation. Applied optics 34, 904-908 (1995).

6. Chubar, O. \& Elleaume, P. Accurate and efficient computation of synchrotron radiation in the near field region. In proc. of the EPAC98 Conference, 1177-1179 (1998).

7. Walker, R. Near field effects in off-axis undulator radiation. Nuclear Instruments and Methods in Physics Research Section A: Accelerators, Spectrometers, Detectors and Associated Equipment 267, 537-546 (1988).

8. Walker, R. P. Interference effects in undulator and wiggler radiation sources. Nuclear Instruments and Methods in Physics Research Section A: Accelerators, Spectrometers, Detectors and Associated Equipment 335, 328-337 (1993).

9. Onuki, H. \& Elleaume, P. Undulators, wigglers and their applications (CRC Press, 2003).

10. Kim, K.-J. Brightness, coherence and propagation characteristics of synchrotron radiation. Nuclear Instruments and Methods in Physics Research Section A: Accelerators, Spectrometers, Detectors and Associated Equipment 246, 71-76 (1986).

11. Geloni, G., Saldin, E., Schneidmiller, E. \& Yurkov, M. Transverse coherence properties of X-ray beams in third-generation synchrotron radiation sources. Nuclear Instruments and Methods in Physics Research Section A: Accelerators, Spectrometers, Detectors and Associated Equipment 588, 463-493 (2008).

12. Giannessi, L. et al. Superradiant cascade in a seeded free-electron laser. Physical review letters 110, 044801 (2013).

13. Lambert, G. et al. Injection of harmonics generated in gas in a free-electron laser providing intense and coherent extreme-ultraviolet light. Nature physics 4, 296 (2008).

14. Tanikawa, T. et al. Nonlinear harmonic generation in a free-electron laser seeded with high harmonic radiation. EPL (Europhysics Letters) 94, 34001 (2011).

15. Desjardins, K. et al. The DiagOn: An undulator diagnostic for SOLEIL low energy beamlines. In Nuclear Science Symposium Conference Record, 2008. NSS'08. IEEE, 2571-2574 (IEEE, 2008).

16. Eriksson, M., van der Veen, J. F. \& Quitmann, C. Diffraction-limited storage rings-a window to the science of tomorrow. Journal of synchrotron radiation $21,837-842$ (2014).

17. Emma, P. et al. First lasing and operation of an ångstrom-wavelength free-electron laser. nature photonics 4, 641 (2010).

18. Madey, J. M. Stimulated emission of Bremsstrahlung in a periodic magnetic field. Journal of Applied Physics 42, 1906-1913 (1971).

19. Gorkhover, T. et al. Femtosecond $\mathrm{x}$-ray Fourier holography imaging of free-flying nanoparticles. Nature Photonics 12, 150 (2018).

20. Tajima, T. \& Dawson, J. M. Laser Electron Accelerator. Phys. Rev. Lett. 43, 267-270, https://doi.org/10.1103/PhysRevLett.43.267. (1979).

21. Leemans, W. P. et al. GeV electron beams from a centimetre-scale accelerator. Nature physics 2, 696 (2006).

22. Leemans, W. P. et al. Multi-GeV Electron Beams from Capillary-Discharge-Guided Subpetawatt Laser Pulses in the Self-Trapping Regime. Phys. Rev. Lett. 113, 245002, https://doi.org/10.1103/PhysRevLett.113.245002 (2014).

23. Faure, J. et al. A laser-plasma accelerator producing monoenergetic electron beams. Nature 431, 541 (2004).

24. Geddes, C. et al. High-quality electron beams from a laser wakefield accelerator using plasma-channel guiding. Nature 431, 538-541 (2004).

25. Mangles, S. et al. Monoenergetic beams of relativistic electrons from intense laser-plasma interactions. Nature 431, 538-541 (2004).

26. Esarey, E., Schroeder, C. \& Leemans, W. Physics of laser-driven plasma-based electron accelerators. Reviews of Modern Physics 81, 1229 (2009).

27. Malka, V. Laser plasma accelerators. Physics of Plasmas 19, 055501, https://doi.org/10.1063/1.3695389 (2012).

28. Chen, M., Sheng, Z.-M., Ma, Y.-Y. \& Zhang, J. Electron injection and trapping in a laser wakefield by field ionization to high-charge states of gases. Journal of Applied Physics 99, 056109 (2006).

29. Gonsalves, A. et al. Petawatt laser guiding and electron beam acceleration to $8 \mathrm{GeV}$ in a laser-heated capillary discharge waveguide. Physical review letters 122, 084801 (2019).

30. Fritzler, S. et al. Emittance measurements of a laser-wakefield-accelerated electron beam. Physical review letters 92, 165006 (2004).

31. Barber, S. et al. Measured emittance dependence on the injection method in laser plasma accelerators. Physical review letters 119, 104801 (2017).

32. Lundh, O. et al. Few femtosecond, few kiloampere electron bunch produced by a laser-plasma accelerator. Nature Physics 7, 219 (2011).

33. Li, Y. et al. Generation of $20 \mathrm{kA}$ electron beam from a laser wakefield accelerator. Physics of Plasmas 24, 023108 (2017).

34. Couperus, J. et al. Demonstration of a beam loaded nanocoulomb-class laser wakefield accelerator. Nature communications 8, 487 (2017).

35. Wang, X. et al. Quasi-monoenergetic laser-plasma acceleration of electrons to $2 \mathrm{GeV}$. Nature communications 4, 1988 (2013).

36. Tsai, H.-E. et al. Control of quasi-monoenergetic electron beams from laser-plasma accelerators with adjustable shock density profile. Physics of Plasmas 25, 043107 (2018).

37. Floettmann, K. Some basic features of the beam emittance. Physical Review Special Topics-Accelerators and Beams 6, 034202 (2003).

38. Migliorati, M. et al. Intrinsic normalized emittance growth in laser-driven electron accelerators. Physical Review Special TopicsAccelerators and Beams 16, 011302 (2013).

39. Antici, P. et al. Laser-driven electron beamlines generated by coupling laser-plasma sources with conventional transport systems. Journal of Applied Physics 112, 044902 (2012).

40. Grüner, F. et al. Design considerations for table-top, laser-based vuv and x-ray free electron lasers. Applied Physics B 86, 431-435 (2007).

41. Nakajima, K. Compact x-ray sources: Towards a table-top free-electron laser. Nature physics 4, 92 (2008)

42. Walker, P. A. et al. Horizon 2020 eupraxia design study. In Journal of Physics: Conference Series, vol. 874, 012029 (IOP Publishing, 2017).

43. Maier, A. et al. Demonstration scheme for a laser-plasma-driven free-electron laser. Physical Review X 2, 031019 (2012).

44. Couprie, M.-E., Loulergue, A., Labat, M., Lehe, R. \& Malka, V. Towards a free electron laser based on laser plasma accelerators. Journal of Physics B: Atomic, Molecular and Optical Physics 47, 234001 (2014).

45. Loulergue, A. et al. Beam manipulation for compact laser wakefield accelerator based free-electron lasers. New Journal of Physics 17, 023028 (2015).

46. Widmann, C. et al. First tests of a beam transport system from a laser wakefield accelerator to a transverse gradient undulator (2015).

47. Liu, T., Zhang, T., Wang, D. \& Huang, Z. Compact beam transport system for free-electron lasers driven by a laser plasma accelerator. Physical Review Accelerators and Beams 20, 020701 (2017).

48. André, T. et al. Control of laser plasma accelerated electrons for light sources. Nature Communications 9, 1334 (2018).

49. Huang, Z., Ding, Y. \& Schroeder, C. B. Compact X-ray free-electron laser from a laser-plasma accelerator using a transverse-gradient undulator. Physical review letters 109, 204801 (2012).

50. Schlenvoigt, H.-P. et al. A compact synchrotron radiation source driven by a laser-plasma wakefield accelerator. Nature Physics 4 , $130-133$ (2008).

51. Fuchs, M. et al. Laser-driven soft-X-ray undulator source. Nature physics 5, 826-829 (2009).

52. Anania, M. P. et al. An ultrashort pulse ultra-violet radiation undulator source driven by a laser plasma wakefield accelerator. Applied Physics Letters 104, 264102 (2014).

53. Lambert, G. et al. Progress on the generation of undulator radiation in the UV from a plasma-based electron beam. In Proceed. FEL conf., Nara, Japan, 2 (2012). 
54. Powers, N. D. et al. Quasi-monoenergetic and tunable X-rays from a laser-driven Compton light source. Nature Photonics 8, 28 (2014).

55. Geddes, C. G. et al. Compact quasi-monoenergetic photon sources from laser-plasma accelerators for nuclear detection and characterization. Nuclear Instruments and Methods in Physics Research Section B: Beam Interactions with Materials and Atoms 350, 116-121 (2015).

56. Schleede, S. et al. Emphysema diagnosis using X-ray dark-field imaging at a laser-driven compact synchrotron light source. Proceedings of the National Academy of Sciences 109, 17880-17885 (2012).

57. Bech, M. et al. Hard X-ray phase-contrast imaging with the Compact Light Source based on inverse Compton X-rays. Journal of synchrotron radiation 16, 43-47 (2009).

58. Couprie, M.-E. et al. An application of laser plasma acceleration: towards a free-electron laser amplification. Plasma Physics and Controlled Fusion 58, 034020 (2016)

59. Marteau, F. et al. Variable high gradient permanent magnet quadrupole (QUAPEVA). Applied Physics Letters 111, 253503 (2017).

60. Emma, P. et al. Femtosecond and Subfemtosecond X-Ray Pulses from a Self-Amplified Spontaneous-Emission21Based FreeElectron Laser. Phys. Rev. Lett. 92, 074801, https://doi.org/10.1103/PhysRevLett.92.074801 (2004).

61. Scisciò, M., Migliorati, M., Palumbo, L. \& Antici, P. Design and optimization of a compact laser-driven proton beamline. Scientific Reports 8, 6299 (2018).

62. Zhu, X., Broemmelsiek, D. R. \& Shin, Y.-M. Theoretical and numerical analyses of a slit-masked chicane for modulated bunch generation. Journal of Instrumentation 10, P10042 (2015).

63. Benabderrahmane, C. et al. Development and operation of a $\operatorname{Pr}_{2} \mathrm{Fe}_{14} \mathrm{~B}$ based cryogenic permanent magnet undulator for a high spatial resolution x-ray beam line. Physical Review Accelerators and Beams 20, 033201 (2017).

64. Chubar, O. et al. Phase Analysis and Focusing of Synchrotron Radiation. Nucl. Instr. and Meth. A435, 495-508 (1999).

65. Valléau, M. et al. Development of cryogenic permanent magnet undulators at SOLEIL. Synchrotron Radiation News 31, 42-47 (2018).

66. Divall, M. Lasers in FEL facilities. CERN Yellow Reports: School Proceedings 1, 327 (2018).

67. Huang, Z. et al. Brightness and coherence of synchrotron radiation and FELs. Tech. Rep., SLAC National Accelerator Lab., Menlo Park, CA (United States) (2013)

68. Molodozhentsev, A. \& Pribyl, L. ELI electron beam line for laser-plasma-driven undulator X-ray source (2016)

69. Ghaith, A. et al. Tunable High Gradient Quadrupoles For A Laser Plasma Acceleration Based FEL. Nuclear Inst. and Methods in Physics Research A 909, 290-293 (2018).

70. Benabderrahmane, C., Couprie, M., Forest, F. \& Cosson, O. Multi - pôle magnétique réglable URL, http://bases-brevets.inpi.fr/fr/ document/WO2016034490.html? p=5 s=1492741420047 cHash=da6678adbfdf5b95b568358d33c57625. WO2016034490 - 201603-10 (2016)

71. Benabderrahmane, C., Couprie, M., Forest, F. \& Cosson, O. Adjustable magnetic multipole, https://patentscope.wipo.int/search/en/ detail.jsf?docId=WO2016034490. Europe: PCT/EP2015/069649 of 27/08/2015, WOBL14SSOQUA/CA. (2016).

72. Le Bec, G., Chavanne, J. \& Penel, C. Stretched wire measurement of multipole accelerator magnets. Physical Review Special TopicsAccelerators and Beams 15, 022401 (2012).

73. Payet, J. et al. Beta code. CEA, SACLAY (2001).

74. Khojoyan, M. et al. Transport studies of LPA electron beam towards the FEL amplification at COXINEL. Nuclear Instruments and Methods in Physics Research Section A: Accelerators, Spectrometers, Detectors and Associated Equipment 829, 260-264 (2016).

75. Chubar, O., Elleaume, P. \& Chavanne, J. A three-dimensional magnetostatics computer code for insertion devices. Journal of synchrotron radiation $5,481-484$ (1998).

76. Polyanskiy, M. N. Refractive index database, https://refractiveindex.info.

77. Shen, Q. X-ray flux, brilliance and coherence of the proposed Cornell energy-recovery synchrotron source, CHESS Technical Memo 01-002 (2001).

\section{Acknowledgements}

This work was partially supported by the FP7 Ideas: European Research Council for the Advanced Grants COXINEL (340015, PI: M.-E. Couprie) and X-Five (339128, PI: V. Malka), the EuPRAXIA design study (653782), the Fondation de la Coopération Scientifique (QUAPEVA-2012-058T), the Agence Nationale de la Recherche (ANR-10-LABX-0039-PALM, LABEX CEMPI ANR-11-LABX-0007), Ministry of Higher Education and Research, Hauts de France council and European Regional Development Fund (Contrat de Projets Etat-Région CPER Photonics for Society P4S). The authors acknowledge J. Daillant, A. Nadji, A. Taleb and A. Rousse for their support. The authors thank A. Thompson and S. Podgorny for English check. The authors would like also to thank members of the Accelerator and Engineering Division and of the Experimental Division of SOLEIL for their help, J. L. Lancelot and his team at Sigmaphi for the joint development of the QUAPEVA magnets.

\section{Author contributions}

S.C., J.G., J.P.G., O. K. G.L., B.M., V.M., S. Se., S.Sm., A.T., C.T. worked on the laser based electron acceleration. The optics was designed by A. Lo., simulations of the transport were done by T. A., A. Lo, D.O. and E.R. Undulator radiation was modelised by A.G., O.C. with the help of M.E.C., O.M., M.L. Equipments were prepared by C. Be., P.B., F.Bl., F. Bo, F.Br, M.E.C., Y.D., A.G., C.K., A. Lo, F.M., M.V., J.V. for the magnetic elements and the undulator, T.A., M.E.C., M.E.A., D.D., N.H., G.L., A. Lo, M.L., F.P., C.T. for the diagnostics, with in particular T.A., A.G., D.D., M.L., D.O., F.P., E.R. for the photon spectrometer and S.C., D.O., S.S., E.R., C.T. for the electron spectrometer. A.C., K.T., C.D.O. worked on the mechanical design and assembly, J.P.D., C.H., P. Rommeluère on the vacuum, T.A., C. Bo., M.E.C., M.L., A. Le, F.M., M.S., M.V. on the alignment, T.A., I.A.A., F. Bl., F. Br, L.C., C.E., M.L., N.L., A. Lo, E.R., M.V. on the control system and high level application. T.A., I.A.A., S.C., M.E.C., M.E.A., J.G., J.P.G., A.G., N.H., O.K., A. Lo, G.L., D.O., E.R., S. Sm., C.T. worked on the experiment. Data were analysed by M.E.C., A.G., E.R., S.B., C.S., C.E. (undulator radiation), T.A., S.C., M.E.C., A. Lo, D.O., E.R., S. Sm., S. Se. (electron beam), T.A., E.C., A. Lo, D.O., E.R. (beam transport). The paper was written by M.E.C., A.G., D.O., S.B.E.R.

\section{Competing interests}

The authors declare no competing interests. 
Additional information

Correspondence and requests for materials should be addressed to A.G.

Reprints and permissions information is available at www.nature.com/reprints.

Publisher's note Springer Nature remains neutral with regard to jurisdictional claims in published maps and institutional affiliations.

(c) (i) Open Access This article is licensed under a Creative Commons Attribution 4.0 International License, which permits use, sharing, adaptation, distribution and reproduction in any medium or format, as long as you give appropriate credit to the original author(s) and the source, provide a link to the Creative Commons license, and indicate if changes were made. The images or other third party material in this article are included in the article's Creative Commons license, unless indicated otherwise in a credit line to the material. If material is not included in the article's Creative Commons license and your intended use is not permitted by statutory regulation or exceeds the permitted use, you will need to obtain permission directly from the copyright holder. To view a copy of this license, visit http://creativecommons.org/licenses/by/4.0/.

(C) The Author(s) 2019 